\title{
AN EXAMPLE OF A FAKE $s$-MANIFOLD WITH A NICE LOCALLY CONTRACTIBLE COMPACTIFICATION
}

\author{
PHILIP L. BOWERS ${ }^{1}$
}

\begin{abstract}
An example is constructed of a topologically complete separable AR $X$ that satisfies the discrete $n$-cells property for each nonnegative integer $n$ but fails to satisfy the discrete approximation property and be homeomorphic to $s$ even though $X$ arises as the complement of a $\sigma$ - $Z$-set in a locally contractible compactum. Such examples are not possible in the setting of ANR compactifications.
\end{abstract}

1. Introduction. The purpose of this note is to present a simple example of a fake $s$-manifold that shows that the main result of [Bow $]$ cannot be generalized from the setting of absolute neighborhood retracts (ANR) to the setting of locally contractible spaces. The main result of $\left[\right.$ Bow $\left._{1}\right]$ is that a space $X$ satisfying the discrete $n$-cells property for each nonnegative integer $n$ is equivalent to the space satisfying the discrete approximation property, provided $X$ arises as the complement of a $\sigma-Z$-set in a locally compact separable ANR. That some extra hypothesis on $X$ is necessary is shown by examples constructed in [BBMW] of topologically complete separble ANR's that satisfy the discrete $n$-cells property for each nonnegative integer $n$ yet fail to satisfy the discrete approximation property and fail to be $s$-manifolds. We apply the technique developed in [BBMW] to construct our example, the starting point of which is Borsuk's construction [Bor, Hu] of a locally contractible compactum that is not an ANR.

A map is a continuous function and $\mathrm{id}_{X}$ denotes the identity map on a space $X$. A closed subset $A$ of a separable metric space $X$ is a $Z$-set in $X$, provided for every open cover $\mathcal{U}$ of $X$ there exists a map $\alpha: X \rightarrow X-A \mathcal{U}$-close to id ${ }_{X}$. The subset $A$ is a strong-Z-set, provided, in addition, the map $\alpha$ can be chosen so that the image of $\alpha$ misses a neighborhood of $A$. If $X$ happens to be locally compact as well, then $Z$-sets are always strong- $Z$-sets; however, this is not true in general [BBMW]. A countable union of $Z$-sets is called a $\sigma-Z$-set. For a nonnegative integer $n$, a space $X$ is said to satisfy the discrete $n$-cells property if for each countable family of maps $f_{i}: I^{n} \rightarrow X, i=1,2, \ldots$, of the $n$-cell to $X$ and open cover $\mathcal{U}$ of $X$, there are $\mathcal{U}$-approximations $g_{i}: I^{n} \rightarrow X, i=1,2, \ldots$, such that the collection $\left\{g_{i}\left(I^{n}\right)\right\}_{i=1}^{\infty}$ is discrete (each point in $X$ has a neighborhood that meets at most one member of the collection). A space $X$ is said to satisfy the discrete approximation property if for each countable family of maps $f_{i}: I^{\infty} \rightarrow X, i=1,2, \ldots$, of the Hilbert cube to $X$ and open cover $\mathcal{U}$ of $X$, there are $\mathcal{U}$-approximations $g_{i}: I^{\infty} \rightarrow X, i=1,2, \ldots$, such that the collection $\left\{g_{i}\left(I^{\infty}\right)\right\}_{i=1}^{\infty}$ is discrete. For the importance of the discrete

Received by the editors July 30, 1985.

1980 Mathematics Subject Classification (1985 Revision). Primary 57N20; Secondary 54B17.

Key words and phrases. Fake s-manifold, Z-set, compactification.

${ }^{1}$ Research partially supported by a summer research grant from The Florida State University and by ONR Grant N00014-84-K-0761. 
properties in the topology of $s$-manifolds (manifolds modeled on $s$, the countably infinite product of open intervals $(0,1))$, see [BBMW, To, Bow 1 ].

I am indebted to Tadeusz Dobrowolski for asking me whether or not the main result of $\left[\right.$ Bow $\left._{1}\right]$ holds in the setting in which he was working, that of locally contractible spaces. Also, I express my sincere appreciation to Doug Curtis for his (as always) helpful advice and suggestions.

2. The example. For a point $x$ in the Hilbert cube $I^{\infty}=\prod_{i=1}^{\infty}[0,1]_{i}, x(i)$ denotes the $i$ th coordinate of $x$. Let $B_{\infty}=\left\{x \in I^{\infty} \mid x(1)=0\right\}$, which is a homeomorphic copy of the Hilbert cube, and for each positive integer $k$ let $B_{k}$ be the $k$-cube contained in $I^{\infty}$ that consists of the points $x \in I^{\infty}$ that satisfy

$$
1 /(k+1) \leq x(1) \leq 1 / k, \quad x(i)=0 \quad \text { for } i>k .
$$

Define subspaces $C$ and $\partial C$ of $I^{\infty}$ as follows:

$$
C=B_{\infty} \cup B_{1} \cup B_{2} \cup \cdots, \quad \partial C=\partial B_{1} \cup \partial B_{2} \cup \cdots,
$$

where $\partial B_{k}$ denotes the boundary $(k-1)$-sphere of $B_{k}$. The subspace $B_{\infty} \cup \partial C$ is Borsuk's example of a locally contractible compactum that is not an ANR [Bor, Hu]. Let $D$ be the following subspace of $I^{\infty} \times[0,1]$ :

$$
D=(C \times\{0\}) \cup(\partial C \times[0,1]) .
$$

$D$ is a topologically complete separable AR (by [KL or $\mathbf{H y}]$ ) and $B=B_{\infty} \times\{0\}$ is a $Z$-set in $D$. In fact, the same arguments used in [BBMW, §5] show that there is an instantaneous deformation of $D$ into $D-B$ and that $B$, though a $Z$-set, is not a strong- $Z$-set in $D$.

Observe that $D$ has a locally contractible compactification, namely $\bar{D}=D \cup$ $\left(B_{\infty} \times[0,1]\right)$, and the difference $\bar{D}-D=B_{\infty} \times(0,1]$ is a $\sigma$-Z-set in $\bar{D}$. The latter part of the previous statement follows since there are small retractions of $C$ onto subsets of the form $B_{k} \cup B_{k-1} \cup \cdots \cup B_{1}$ that restrict to retractions of $B_{\infty} \cup \partial C$ onto $\left(B_{k} \cup B_{k-1} \cup \cdots \cup B_{1}\right) \cap \partial C$.

The example referred to in the Introduction is gotten by taking the product of $D$ and $s$ reduced about $B$, denoted $(D \times s)_{B} .(D \times s)_{B}$ is the set $[(D-B) \times s] \cup B$ equipped with the topology generated by open subsets of $(D-B) \times s$ and sets of the form $((U-B) \times s) \cup(U \cap B)$, where $U \subset D$ is open. $(D \times s)_{B}$ is a topologically complete separable AR [BBMW, $\S 1]$, and $B$ is a $Z$-set in $(D \times s)_{B}$ but not a strong- $Z$-set [BBMW, Corollary 1.2]. It then follows from [Bow 2 , Lemma 1, §4] that $(D \times s)_{B}$ does not have a nice ANR local compactification in the sense that $(D \times s)_{B}$ does not arise as the complement of a $\sigma$-Z-set in a locally compact ANR; however, $(D \times s)_{B}$ does have a nice locally contractible compactification.

2.1. EXAMPLE. $(D \times s)_{B}$ is a topologically complete separable AR that satisfies the discrete $n$-cells property for each nonnegative integer $n$ but fails to satisfy the discrete approximation property. However, $(D \times s)_{B}$ has a nice locally contractible compactification in the sense that $(D \times s)_{B}$ is the complement of a $\sigma-Z$-set in a locally contractible compactum.

For the proof of the claims of the example, we need the following lemma, whose proof involves a straightforward construction and is left as an exercise for the reader. 
2.2. LEMMA. Let $\alpha$ and $\beta$ be positive integers and $0<t<1 / 2$. Define an $\alpha$-cell $J_{t}^{\alpha}$ contained in the $(\alpha+\beta)$-cell $I^{\alpha+\beta}=[0,1]_{1} \times \cdots \times[0,1]_{\alpha+\beta}$ by

$$
J_{t}^{\alpha}=[t, 1-t]_{1} \times \cdots \times[t, 1-t]_{\alpha} \times\{1 / 2\}_{\alpha+1} \times \cdots \times\{1 / 2\}_{\alpha+\beta} .
$$

Then there exists a retraction $r:\left(I^{\alpha+\beta}-J_{t}^{\alpha}\right) \rightarrow \partial I^{\alpha+\beta}$ such that $r$ moves the last $\beta$ coordinates freely while moving the first $\alpha$ coordinates by no more than $t$. More precisely, let $p_{i}: I^{\alpha+\beta} \rightarrow[0,1]_{i}$ be the ith coordinate projection. Then $\mid p_{i}(x)-$ $p_{i}(r(x)) \mid \leq t$ for each point $x$ in $I^{\alpha+\beta}-J_{t}^{\alpha}$ and each $i \in\{1, \ldots, \alpha\}$.

ProOF OF 2.1. First, if $(D \times s)_{B}$ satisfies the discrete approximation property, then $Z$-sets are strong- $Z$-sets [BBMW, Proposition 1.3], contradicting the fact that the $Z$-set $B$ is not a strong- $Z$-set in $(D \times s)_{B}$. To show that $(D \times s)_{B}$ satisfies the discrete $n$-cells property for each $n$, it suffices to show that, given a positive number $\varepsilon$ and a nonnegative integer $n$, any countable family of maps $f_{1}, f_{2}, \ldots$ of the $n$-cell $I^{n}$ into $D$ has $3 \varepsilon$-approximations $g_{1}, g_{2}, \ldots$ whose images miss a neighborhood of $B$ and for which $f_{i}=g_{i}$ on $f_{i}^{-1}\left(D-N_{\varepsilon}(B)\right)$ for each $i$, where $N_{\varepsilon}(B)$ denotes the $\varepsilon$-neighborhood of $B$ in $D$. Choose a positive integer $m$ so large that, for all $k>m,\left(B_{k} \times\{0\}\right) \cup\left(\partial B_{k} \times[0,1 / m]\right)$ is contained in $N_{\varepsilon}(B)$ and, recalling that $D \subset I^{\infty} \times[0,1]$, so that any move in $D$ that affects only coordinates greater than $m$ moves points at most $\varepsilon$. Since $B$ is a $Z$-set in $D$, we may assume that the image of each $f_{i}$ misses $B$. Fix a positive integer $\alpha>m$ and let $h: B_{\alpha+n+1} \times\{0\} \rightarrow I^{\alpha+n+1}$ be the obvious linear homeomorphism induced by the linear homeomorphism $[1 /(\alpha+n+2), 1 /(\alpha+n+1)] \rightarrow[0,1]$ between the first factors. Choose $t$ so small that if $r$ denotes the retraction of Lemma 2.2 with $\alpha$, $\beta=n+1$, and $t$, then the distance between $x \in B_{\alpha+n+1} \times\{0\}-h^{-1}\left(J_{t}^{\alpha}\right)$ and $h^{-1} \circ \operatorname{roh}(x)$ is less than $2 \varepsilon$. Since $I^{n}$ is $n$-dimensional, we assume that the image of each $f_{i}$ misses $h^{-1}\left(J_{t}^{\alpha}\right)$, and by applying $h^{-1} \circ r \circ h$ we obtain $2 \varepsilon$-approximations $f_{i}^{\prime}$ to $f_{i}$ such that $f_{i}^{\prime}\left(I^{n}\right) \cap B_{\alpha+n+1} \times\{0\}$ is contained in $\partial B_{\alpha+n+1} \times\{0\}$ for each $i$. For a positive integer $k$, let $C_{k}=B_{\infty} \cup B_{k} \cup B_{k+1} \cup \cdots$, and let $\partial C_{k}=C_{k} \cap \partial C$. Letting $\alpha$ range over all positive integers greater than $m$, we obtain $2 \varepsilon$-approximations $g_{i}^{\prime}$ to $f_{i}$ such that $g_{i}^{\prime}\left(I^{n}\right) \cap\left(C_{m+n+2} \times\{0\}\right)$ is contained in $\partial C_{m+n+2} \times\{0\}$. A final move in the $[0,1]$-direction of $D$ produces approximations $g_{i}$ so that for each $i$ the image of $g_{i}$ misses $\left(C_{m+n+3} \times\{0\}\right) \cup\left(\partial C_{m+n+3} \times[0,1 / 2 m]\right)$, a neighborhood of $B$ in $D$.

We now show that $(D \times s)_{B}$ has a nice locally contractible compactification. Recall that $\bar{D}=D \cup\left(B_{\infty} \times[0,1]\right)$. The reduced product $\left(\bar{D} \times I^{\infty}\right)_{B}$ contains $(D \times s)_{B}$ as a dense subspace, and it is easy to show that since $\bar{D}$ is locally contractible, $\left(\bar{D} \times I^{\infty}\right)_{B}$ is a locally contractible compactum. Since $B_{\infty} \times(0,1]$ is a $\sigma$ - $Z$-set in $\bar{D}$ and $B\left(I^{\infty}\right)=I^{\infty}-s$ is a $\sigma$-Z-set in $I^{\infty}$, it follows that

$$
\begin{aligned}
\left(\bar{D} \times I^{\infty}\right)_{B} & -(D \times s)_{B}=(\bar{D}-B) \times I^{\infty}-(D-B) \times s \\
= & \left(\left(B_{\infty} \times(0,1]\right) \times I^{\infty}\right) \cup\left((\bar{D}-B) \times B\left(I^{\infty}\right)\right)
\end{aligned}
$$

is a $\sigma$-Z-set in $\left(\bar{D} \times I^{\infty}\right)_{B}$. The only difficulty is in showing that a set of the form $\left(B_{\infty} \times[t, 1]\right) \times I^{\infty}$ for $0<t<1$ is a $Z$-set in $\left(\bar{D} \times I^{\infty}\right)_{B}$. For an open neighborhood $U$ of $B$ in $\bar{D}$ let $\theta$ be a Urysohn function with $\theta=0$ on $\bar{D}-U$ and $\theta=1$ on $B$, and let $H$ be a contraction of $I^{\infty}$ to a point with $H_{0}=\mathrm{id}_{I^{\infty}}$ and $H_{1}$ constant. Let $p: \bar{D} \times I^{\infty} \rightarrow\left(\bar{D} \times I^{\infty}\right)_{B}$ denote the obvious projection map, and let $r: \bar{D} \rightarrow \bar{D}$ be a 
small map for which $\left(B_{\infty} \times[0,1]\right) \cap r(\bar{D})=\varnothing$. Let $q: \bar{D} \times I^{\infty} \rightarrow \bar{D} \times I^{\infty}$ be the map defined by $q(d, t)=(r(d), H(t, \theta(d)))$ for $(d, t) \in \bar{D} \times I^{\infty}$ and observe that since $p$ is a quotient map and $q \circ p^{-1}$ is single valued, $f=p \circ q \circ p^{-1}$ is a well-defined map of $\left(\bar{D} \times I^{\infty}\right)_{B}$ into $\left(\bar{D} \times I^{\infty}\right)_{B}$ whose image misses $\left(B_{\infty} \times(0,1]\right) \times I^{\infty}$. If $U$ is a small enough neighborhood of $B$ and $r$ is close enough to id $\bar{D}$, then $f$ will be as close to the identity on $\left(\bar{D} \times I^{\infty}\right)_{B}$ as we wish; hence, $\left(B_{\infty} \times[t, 1]\right) \times I^{\infty}$ is a $Z$-set in $\left(\bar{D} \times I^{\infty}\right)_{B}$.

\section{REFERENCES}

[BBMW] M. Bestvina, P. Bowers, J. Mogilski and J. Walsh, Characterization of Hilbert space manifolds revisited, Topology Appl. (to appear).

[Bor] K. Borsuk, Sur un espace compact localement contractile que n'est pas un réstracte absolu de voisinage, Fund. Math. 35 (1948), 175-180.

[Bow 1 ] P. Bowers, Discrete cells properties in the boundary set setting, Proc. Amer. Math. Soc. 93 (1985), 735-740.

[Bow 2 ] _ Nonshrinkable 'cell-like' decompositions of s, Pacific J. Math. (to appear).

[Hu] S. Hu, Theory of retracts, Wayne State Univ. Press, Detroit, Mich., 1965.

[Hy] D. M. Hyman, A generalization of the Borsuk-Whitehead-Hanner theorem, Pacific J. Math. 23 (1967), 263-271.

[KL] A. Kruse and P. Liebnitz, An application of a family homotopy extension theorem to ANR spaces, Pacific J. Math. 16 (1966), 331-336.

[To] H. Torunczyk, Characterizing Hilbert space topology, Fund. Math. 111 (1981), 247-262.

Department of Mathematics, The Florida State University, Tallahassee, FLORIDA 32306-3027 